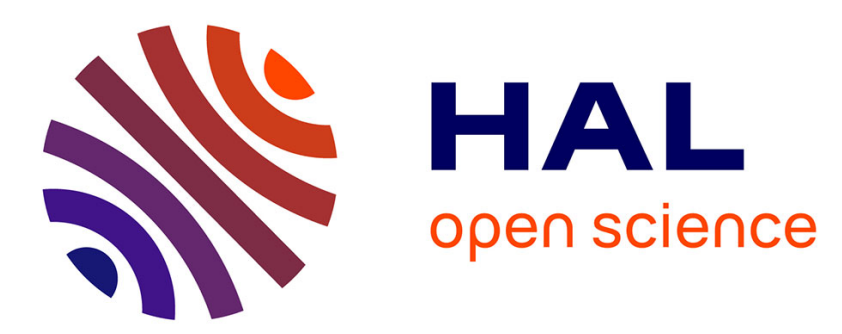

\title{
Analisi e rilettura di alcuni impianti idrici nell'Area Sacra di largo Argentina
}

\author{
Monica Ceci, Alessandra Marchello, Marcello Turci
}

\section{To cite this version:}

Monica Ceci, Alessandra Marchello, Marcello Turci. Analisi e rilettura di alcuni impianti idrici nell'Area Sacra di largo Argentina. Mortiers et hydraulique en Méditerranée antique, Mar 2016, Aix-en-Provence, France. hal-02510813

\section{HAL Id: hal-02510813 \\ https://hal.science/hal-02510813}

Submitted on 18 Mar 2020

HAL is a multi-disciplinary open access archive for the deposit and dissemination of scientific research documents, whether they are published or not. The documents may come from teaching and research institutions in France or abroad, or from public or private research centers.
L'archive ouverte pluridisciplinaire HAL, est destinée au dépôt et à la diffusion de documents scientifiques de niveau recherche, publiés ou non, émanant des établissements d'enseignement et de recherche français ou étrangers, des laboratoires publics ou privés. 


\section{ARCHÉOLOGIES MÉDITERRANÉENNES}

collection dirigée par

Marie-Brigitte Carre et François Quantin

Dans la même collection:

Sophie BOUFFIER, Oscar BELVEDERE et Stefano VASSALLO, dir., Gérer l'eau en Méditerranée au premier millénaire avant J.-C., 230 p., 2019

Emma MAGLIO, Rhodes: forme urbaine et architecture religieuse (XIVe-XVIII siècles), 180 p., 2016

Colette CASTRUCCI, Le quartier du port de Marseille 1500-1790 : une réalité urbaine restituée, 218 p., 2016.

Prix Félix de Beaujour, Académie des Sciences, Lettres et Arts de Marseille

Catherine DUREUIL-BOURACHAU, Archéologie au présent. Les découvertes de l'archéologie préventive dans les médias, 109 p., 2015

Stéphane BOURDIN, Julien DUBOULOZ et Emmanuelle ROSSO, dir., Peupler et habiter l'Italie et le monde romain, 240 p., 2014 


\section{Mortiers}

\section{et hydraulique \\ en Méditerranée antique}

sous la direction de

Iván Fumadó Ortega et Sophie Bouffier 
@ Presses Universitaires de Provence

Aix-Marseille Université

29, avenue Robert-Schuman - F - 13621 Aix-en-Provence CEDEX 1 Tél. 33 (0)4 13553191

pup@univ-amu.fr - Catalogue complet sur http://presses-universitaires.univ-amu.fr

DIFFUSION LIBRAIRIES: AFPU DIFFUSION - DISTRIBUTION SODIS 


\section{Analisi e rilettura di alcuni impianti idrici nell'Area Sacra di largo Argentina}

\author{
Monica $\mathrm{CECl}$ \\ Sovrintendenza Capitolina ai \\ Beni Culturali
}

\author{
Alessandra MARCHELLO \\ Sapienza Università di Roma
}

\author{
Marcello TURCI \\ Aix Marseille Univ, CNRS, CCJ \\ Aix-en-Provence, France
}

\begin{abstract}
Between the 1920s and the 1930s a complex water system, consisting of five elements, has been identified behind the temple B and in the area between temples B and C. Starting from the detailed but confused journal of excavation, verified in the field through a careful reexamination of archaeological data, we propose a new interpretation of these structures using archaeometrical analysis carried out on the mortars and coatings.
\end{abstract}

Già nella primavera del 1927 Giuseppe Marchetti Longhi, che seguiva tra mille difficoltà i lavori di scavo nell'area sacra di largo Argentina, segnalò la presenza di una struttura idrica posta dietro il tempio B (vasca 1$)^{1}$. Nel corso delle convulse operazioni che portarono all'inaugurazione del 21 aprile 1929, ne furono individuate altre quattro (Fig. 1), situate in prossimità della prima, alle spalle del tempio B (vasche 2 e 3), ma anche in connessione con il contropodio del tempio stesso (vasca 5) e nella zona interposta tra il tempio B e il tempio C (vasca 4).

Attraverso l'analisi del Giornale di Scavo di quegli anni, verificato sul campo da un'attenta rilettura dei dati archeologici, è possibile proporre una nuova interpretazione di tali strutture, anche alla luce delle informazioni fornite dalle analisi archeometriche effettuate sulle malte di allettamento e di rivestimento ${ }^{2}$. Lo studio è stato condotto attraverso il rilievo e la puntuale analisi stratigrafica delle opere murarie.

La vasca più antica, indicata nella planimetria (Fig. 1) con il numero 5, presenta una forma rettangolare, ma con il lato interno curvilineo che segue la forma del contropodio del

1 Giornale di Scavo del 23 Aprile 1927, p. 15; i resoconti di scavo, conservati insieme a una grande quantità di materiale documentario (foto, corrispondenza, appunti sui materiali rinvenuti etc.) nell'Archivio Storico della Sovrintendenza Capitolina, sono in corso di trascrizione ed edizione da parte di Monica Ceci. Marchetti Longhi pubblicò il sistema di vasche solo molti anni dopo: Marchetti Longhi 1970-71: p. 26-30; sulle frenetiche attività di scavo e di restauro che condussero all'inaugurazione del 1929, v. Manacorda, Tamassia 1985; Mancioli 1995; Messa 1995; Galluppi 2007.

2 Per lo studio e la caratterizzazione delle malte idrauliche ci si è avvalsi del contributo del CISTeC, il Centro di Ricerche di Scienze e Tecnica per la Conservazione del Patrimonio Storico Archeologico dell'Università Sapienza di Roma, diretto dalla prof.ssa Maria Laura Santarelli, che ringraziamo per la disponibilità e per aver saputo ascoltare e comprendere le nostre richieste, facilitando le nostre ricerche (v. appendice).
Résumé - Entre 1920 et 1930 un complexe hydraulique, constitué d'au moins cinq éléments, a été identifié sur le flanc arrière du temple B de l'Area Sacra de Largo Argentina et dans le secteur entre le temple B et le temple C. En partant des journaux de fouille de ces années, détaillés mais touffus, et après des vérifications de terrain obtenues grâce à un réexamen des données archéologiques, nous proposons une nouvelle interprétation de ces structures en utilisant l'analyse archéométrique des mortiers et enduits.

tempio B. A partire da Marchetti Longhi, tutta la tradizione degli studi l'ha messa in relazione con la prima fase del tempio rotondo, collocabile attorno al 100 a.C., o comunque l'ha considerata anteriore all'ampliamento del tempio stesso, realizzato con la costruzione del primo contropodio ${ }^{3}$. Tuttavia, da un attento esame delle strutture archeologiche attualmente visibili, risulta chiaramente che i lati corti del bacino 5 si appoggiano alla fondazione del contropodio (Fig. 2). Il terminus post quem per la realizzazione della prima vasca è rappresentato, quindi, dalla costruzione del contropodio stesso, la cui datazione è stata inquadrata da Filippo Coarelli nel terzo quarto del I secolo a.C. sulla base dell'analisi stilistica delle modanature e del rinvenimento nel conglomerato di alcuni frammenti di "lastre Campana", difficilmente databili prima della metà dello stesso secolo ${ }^{4}$.

La vasca, conservata in tutta la sua altezza, misura $1,27 \mathrm{~m}$, con una superficie di $5,50 \mathrm{~m}^{2}$ e una capacità stimata di circa $7 \mathrm{~m}^{3}$. La stretta relazione topografica e cronologica con il contropodio e l'assenza di canalizzazioni di alimentazione, fa ipotizzare che il bacino fosse alimentato dalla raccolta delle acque meteoriche provenienti dal tetto del tempio B. La vasca 5 ebbe certamente una vita piuttosto breve dal momento che venne tagliata, in età augustea, dalla vasca $2^{5}$ (Fig. 1). Quest'ultima ha una forma mistilinea a pianta rettangolare terminante in un'abside, si sviluppa per una superficie di $5 \mathrm{~m}^{2}$ ed è conservata per un'altezza massima dal fondo di 0,75 $\mathrm{m}$.

La vasca 3 (Fig. 1), di forma del tutto simile, risulta leggermente più grande, con una superficie di 7,13 $\mathrm{m}^{2}$, ed è conservata per un'altezza massima di m 0,80. Entrambe le vasche sono localizzate a circa un metro dal contropodio del

\footnotetext{
3 Marchetti Longhi 1970-71: 27; Coarelli 1981: 24.

4 Coarelli 1981: 21 .

5 Per la datazione della vasca 2 all'età augustea v. oltre.
} 
Fig. 1 - Area Sacra di largo Argentina. In evidenza gli impianti idrici (Archivio Disegni Sovrintendenza Capitolina - U.O. Monumenti di Roma con rielaborazioni degli autori).

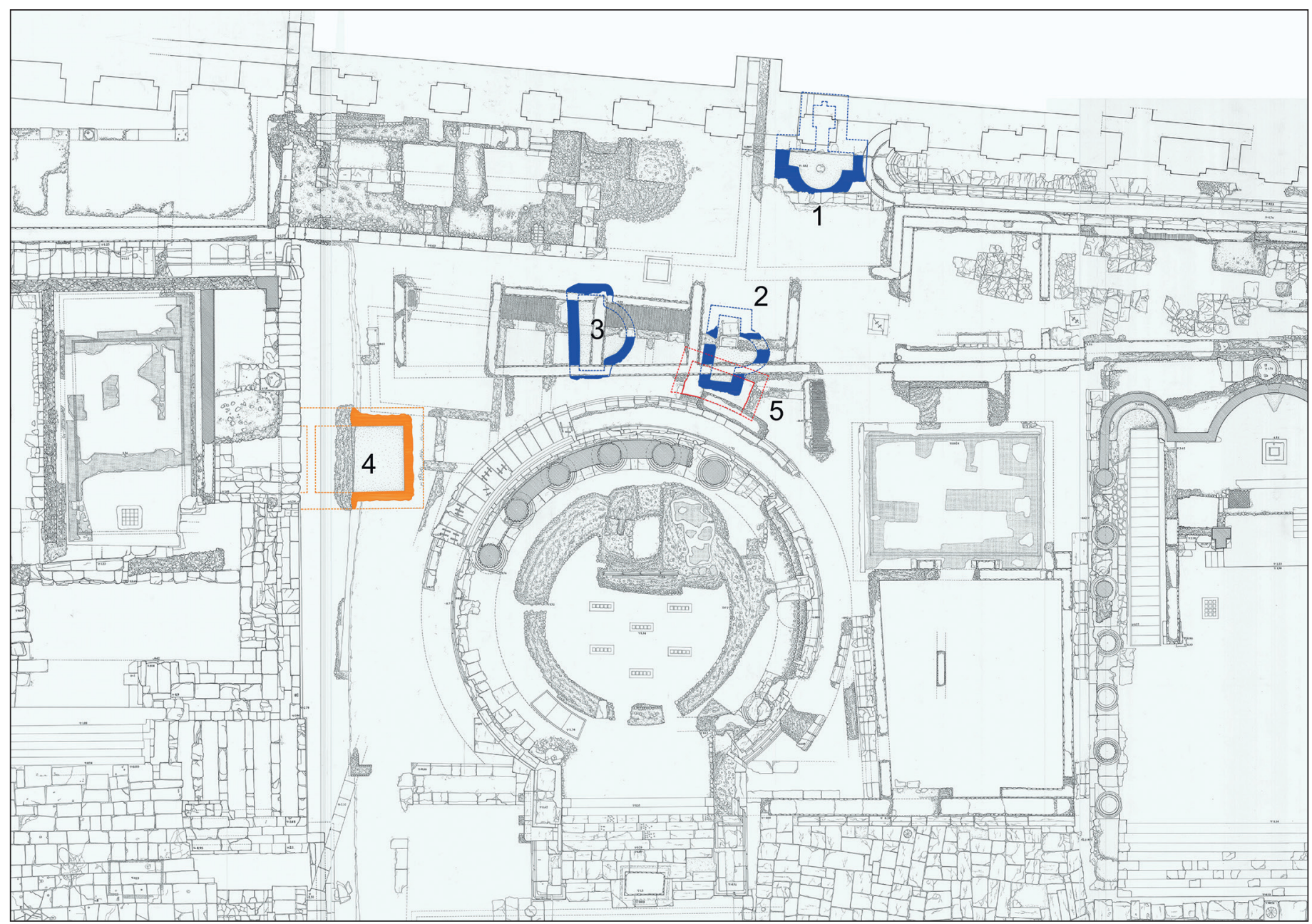

tempio B e presentano un disassamento di $8^{\circ}$ che ne asseconda la circonferenza. Vennero successivamente obliterate da un edificio in opera laterizia, denominato edificio 1 (Fig. 3), caratterizzato da piccoli ambienti collegati da un corridoio di disimpegno ad L e realizzato in appoggio alla parte posteriore del tempio B e al lato nord del tempio C.

La vasca 1 (Fig. 1) è conservata solo parzialmente, poiché la parte occidentale fu distrutta alla fine degli anni '20 durante i lavori di costruzione del muro di terrazzamento dell'area archeologica. Inoltre parte della muratura dell'abside risulta asportata già in antico dal passaggio di una fogna per lo smaltimento delle acque dell'adiacente grande latrina. La forma originaria è comunque nota da uno schizzo misurato di G. Marchetti Longhi, (Fig. 4) in seguito riportato nella pianta generale di G. Ioppolo (Fig. 5). Presenta anch'essa una forma rettangolare con terminazione absidata collegata tramite una sorta di canale ad una seconda vasca rettangolare più piccola. Oltre che per la planimetria più articolata si differenzia dalle precedenti per la presenza di nicchie ricavate nelle pareti: due nell'abside, due laterali e una quinta riportata in pianta e posta sul fondo della vaschetta rettangolare oggi scomparsa (Figg. 6 e 7). Al centro dell'abside doveva trovarsi un elemento, una sorta di scoglio con piccole nicchie non più visibile, ma documentato in una foto d'archivio (Fig. 8) e nella planimetria redatta negli anni 90 (Fig. 1). Le piccole nicchie suggerirono a Marchetti Longhi che la funzione di questa vasca fosse legata alla presenza di pesci ${ }^{6}$. La vasca doveva avere un'estensione totale di circa $6,80 \mathrm{~m}^{2}$ e, a differenza delle due precedenti, è possibile stabilire l'altezza dell'invaso fino al piano di campagna poiché questo si conserva sul lato nord, al di sotto dell'abside della grande latrina. Tale dato permette di stimarne una capacità pari a $8,66 \mathrm{~m}^{3}$.

Da un punto di vista costruttivo le vasche 1, 2 e 3 presentano le medesime caratteristiche sia per quanto riguarda il conglomerato impiegato che per il tipo di rivestimento delle pareti. È quindi plausibile considerarle coeve.

6 Marchetti Longhi 1970-71: 29. 
Fig. 2 - Area Sacra di largo Argentina. Particolare della vasca 5 in cui è visibile che l'impianto idrico si appoggia alla fondazione del contropodio del tempio B.

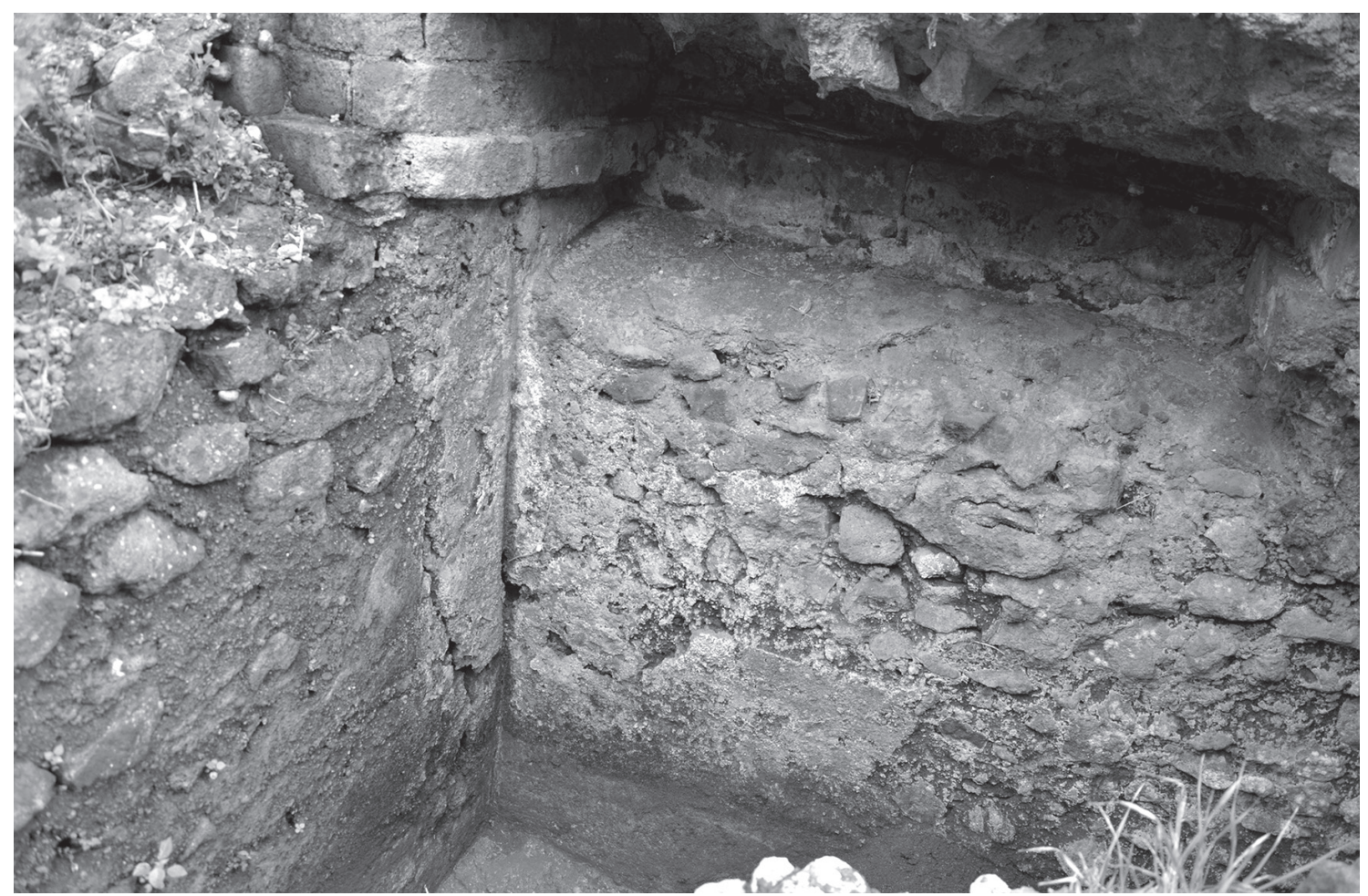

Non si hanno indizi utili a stabilire il sistema di alimentazione che tuttavia doveva essere effettuato tramite fistulae, generalmente poste più in alto rispetto ai livelli conservati ${ }^{7}$. Il foro di deflusso è visibile solamente nel caso della vasca 1 . Infine, l'assenza di opere murarie associabili alle vasche fa ipotizzare che in questa fase la zona fra la Curia e il tempio B fosse relativamente sgombra da edifici e adibita ad area verde.

Il terminus post quem per la cronologia dei bacini mistilinei è rappresentato dalla datazione della vasca 5 nel terzo quarto del I secolo a.C., mentre il terminus ante quem è fornito dalla costruzione dell'edificio 1 alle spalle del tempio B, inquadrabile in una fase precedente al restauro domizianeo dell'area sacra, che obliterò le vasche $1 \mathrm{e} 2^{8}$.

In sintesi, accertato che la vasca 5 è posteriore al primo contropodio del tempio B, datato al terzo quarto del I sec. a.C., e che è obliterata nella fase augustea dalla vasca 2 , risulta plausibile una sua datazione a un momento estremamente vicino, o forse coincidente, con la costruzione del contropodio stesso, che va posto in relazione con i lavori promossi da Pompeo per la realizzazione del grandioso complesso edilizio situato alle spalle dei templi di largo Argentina.

7 Dessales 2013: 207-208.

8 Coarelli ha proposto una datazione dell'edificio 1 intorno alla metà del I secolo d.C. sulla base di un bollo dell'età di Claudio (Coarelli 1981: 24; Steinby 1981: 302), rinvenuto da Marchetti Longhi: "demolendo le strutture lungo il fianco nord del tempio C” (Marchetti Longhi 1970-71:37).
Le vasche 1,2 e 3 , invece, si inquadrano, con ogni probabilità, in età augustea nell'ambito delle opere che, per volere di Agrippa, interessarono il Campo Marzio tra il 25 ed il 19 a.C. Tali interventi portarono tra l'altro alla costruzione dell'Aqua Virgo che con ogni probabilità alimentava i bacini mistilinei.

La vasca 4 , posta tra il tempio B e il tempio $C$, presenta una forma rettangolare con pareti in opera laterizia. Essa è conservata solo parzialmente perché tagliata nel 1929 dagli interventi di A. Muñoz per l'isolamento del podio del tempio $\mathrm{C}^{10}$. Sulla base della documentazione d'archivio si stima che la struttura si sviluppasse su una superficie di $15 \mathrm{~m}^{2}$ e originariamente poggiasse contro il podio del tempio C. In base all'orientamento e ai livelli delle murature si può considerare di pertinenza dell'edificio 2 (Fig. 3) e quindi inquadrare nel corso del II secolo d.C. ${ }^{11}$.

9 Coarelli 1981: 24

10 Il precario stato di conservazione, dovuto al pesante restauro delle cortine e del fondo, non ha permesso di eseguire una campionatura da sottoporre ad analisi archeometriche.

11 Tale datazione, già proposta da G. Lugli (1938: 36, 44), sarà discussa in un articolo in corso di pubblicazione. Contra F. Coarelli che data la struttura all'età di Caracalla (Coarelli 1981: 24). È certa in ogni caso la posteriorità rispetto agli interventi domizianei (cf. Santangeli Valenzani 1994: 68). 
Fig. 3 - Area Sacra di largo Argentina. In evidenza in verde l'edificio 1 e in arancione l'edificio 2 (Archivio Disegni Sovrintendenza Capitolina- U.O. Monumenti di Roma con rielaborazioni degli autori).

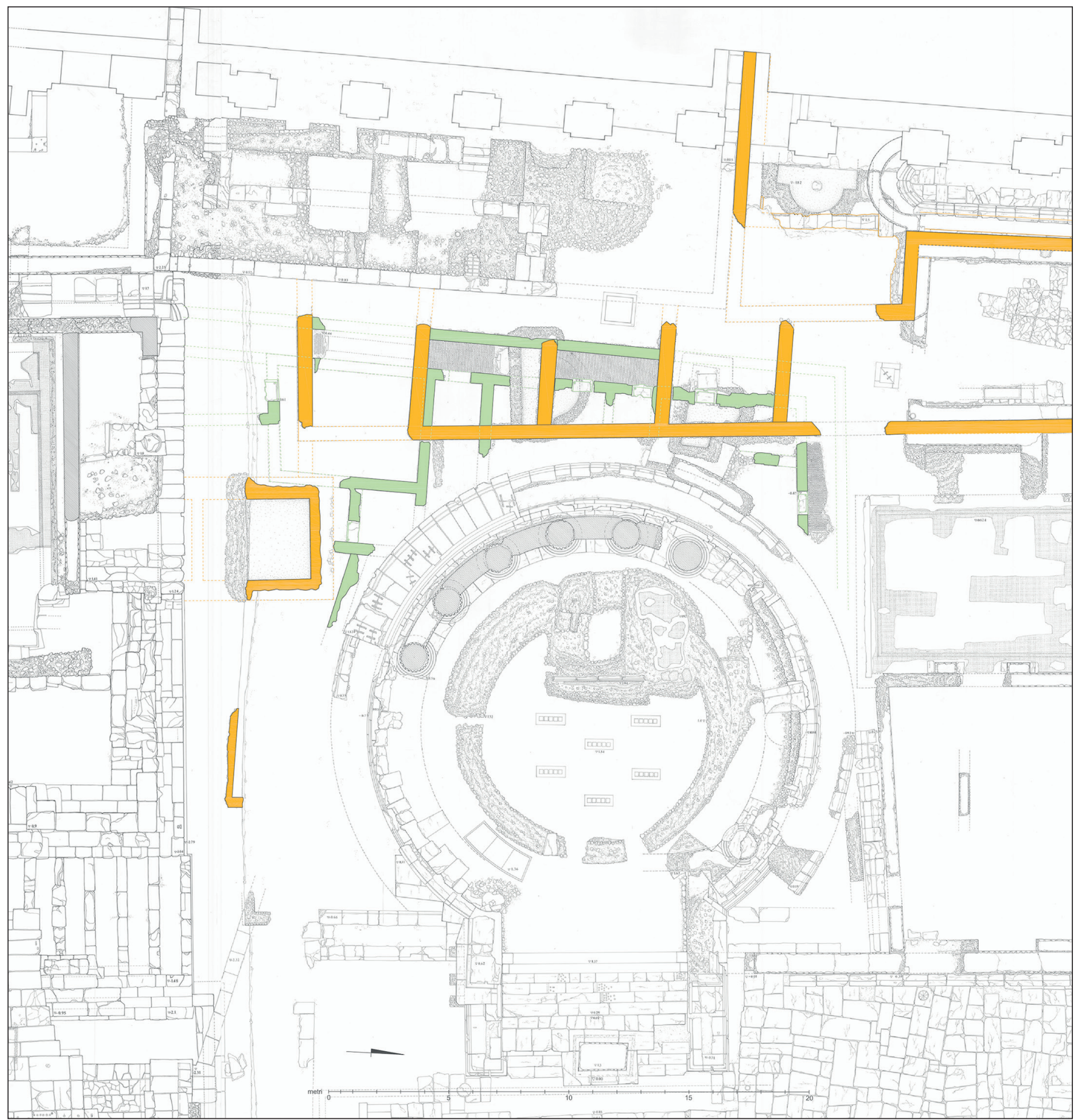


Fig. 4 - Area Sacra di largo Argentina. Stralcio di uno schizzo misurato di G. Marchetti Longhi relativo alla vasca 1 (Archivio Storico Sovrintendenza Capitolina - Giornale di Scavo 1927).

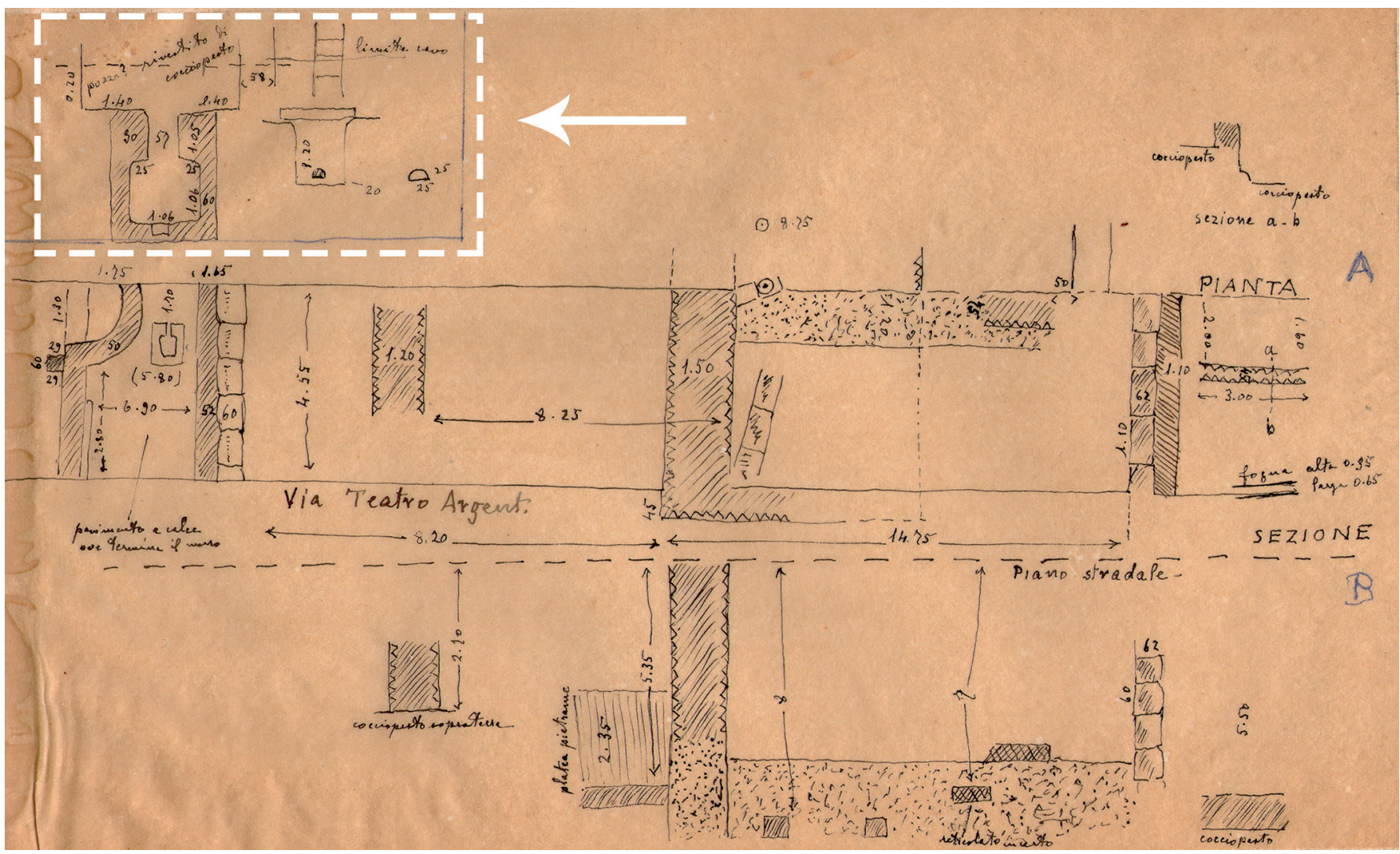

Fig. 5 - Area Sacra di largo Argentina. Planimetria generale dell'area (da Coarelli 1981).

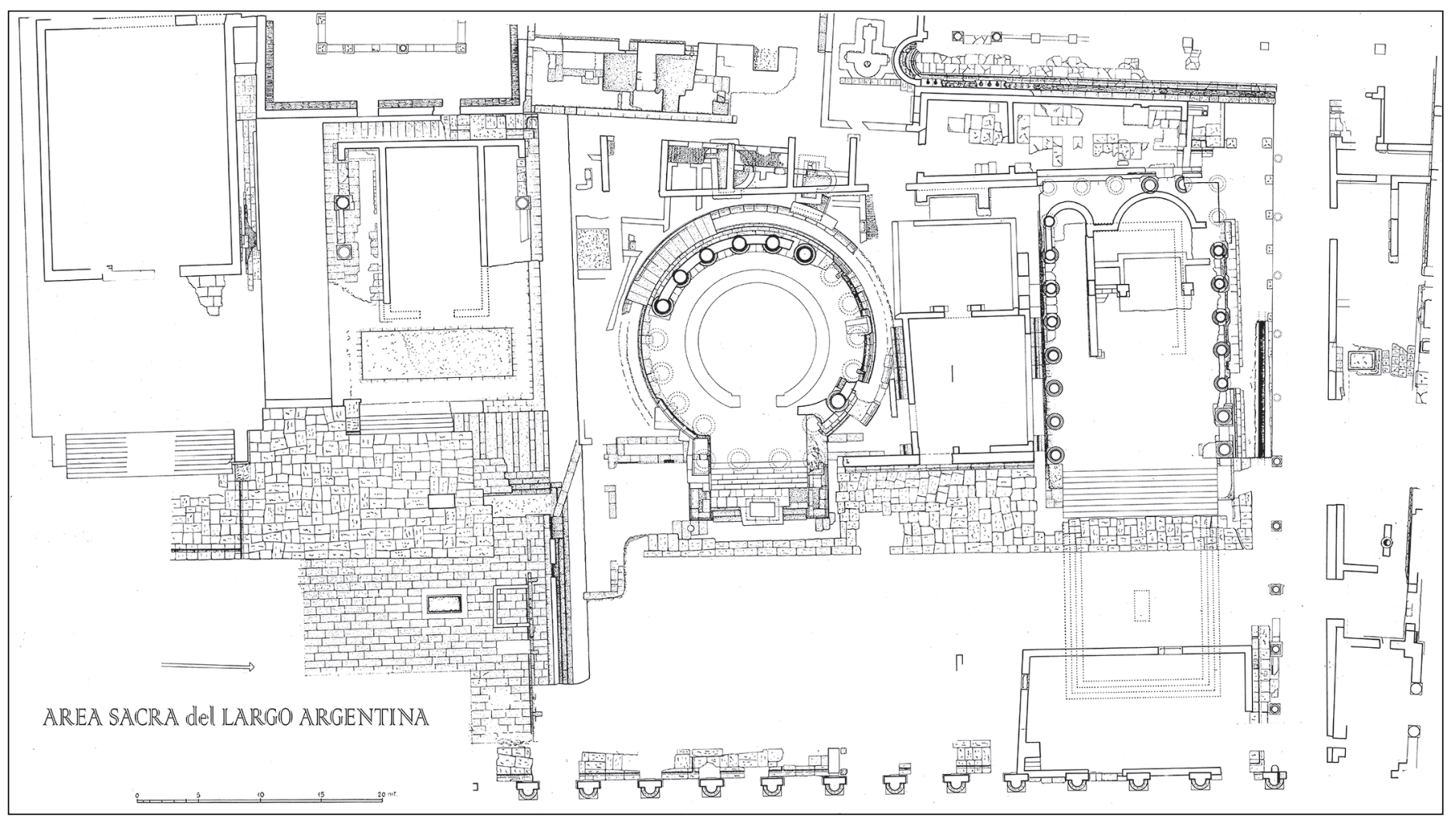


Fig. 6 - Area Sacra di largo Argentina. La vasca 1 tagliata dalla fogna della latrina e dagli interventi moderni.

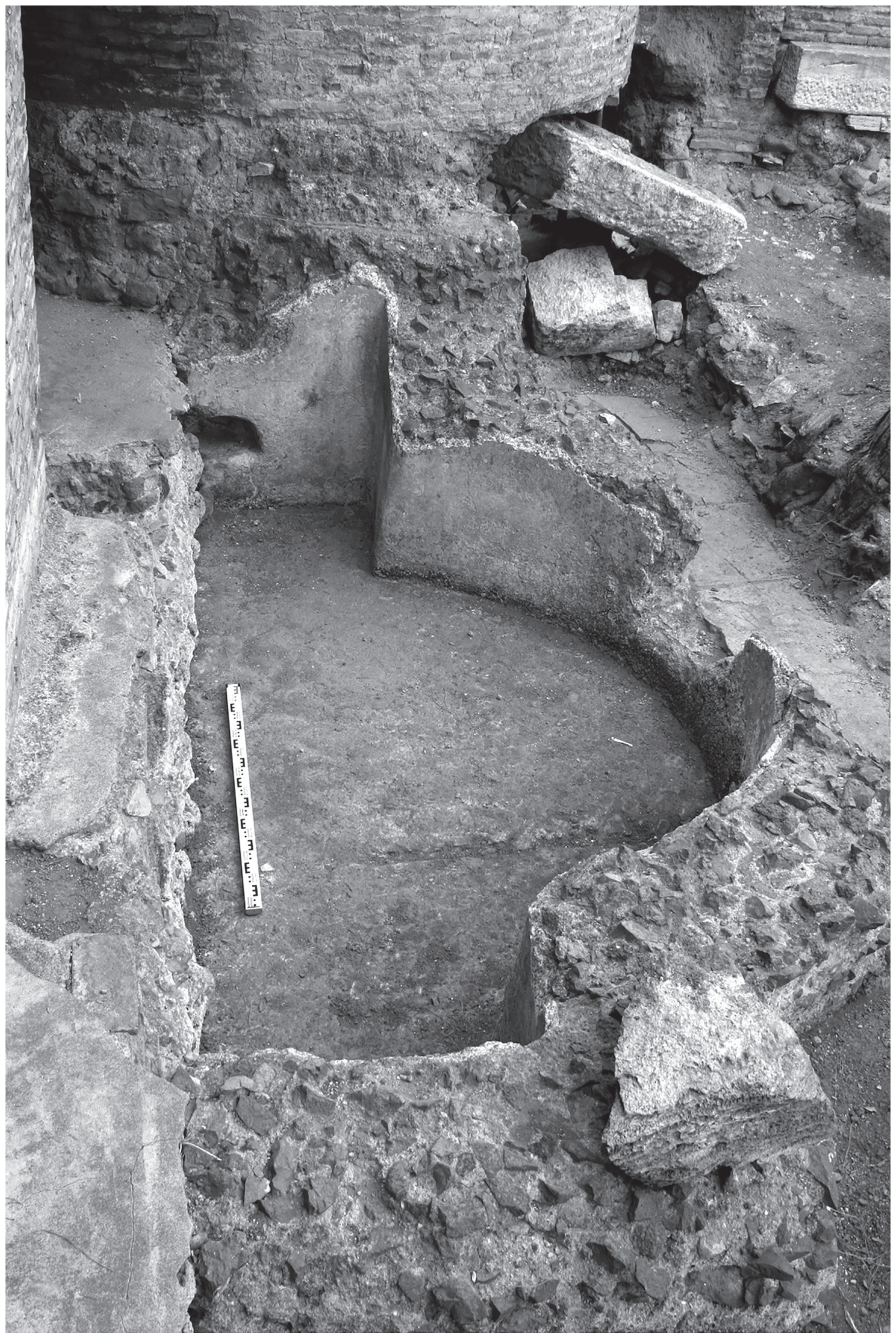


Fig. 7 - Area Sacra di largo Argentina. La vasca 1: particolare delle piccole nicchie ricavate sulle pareti.

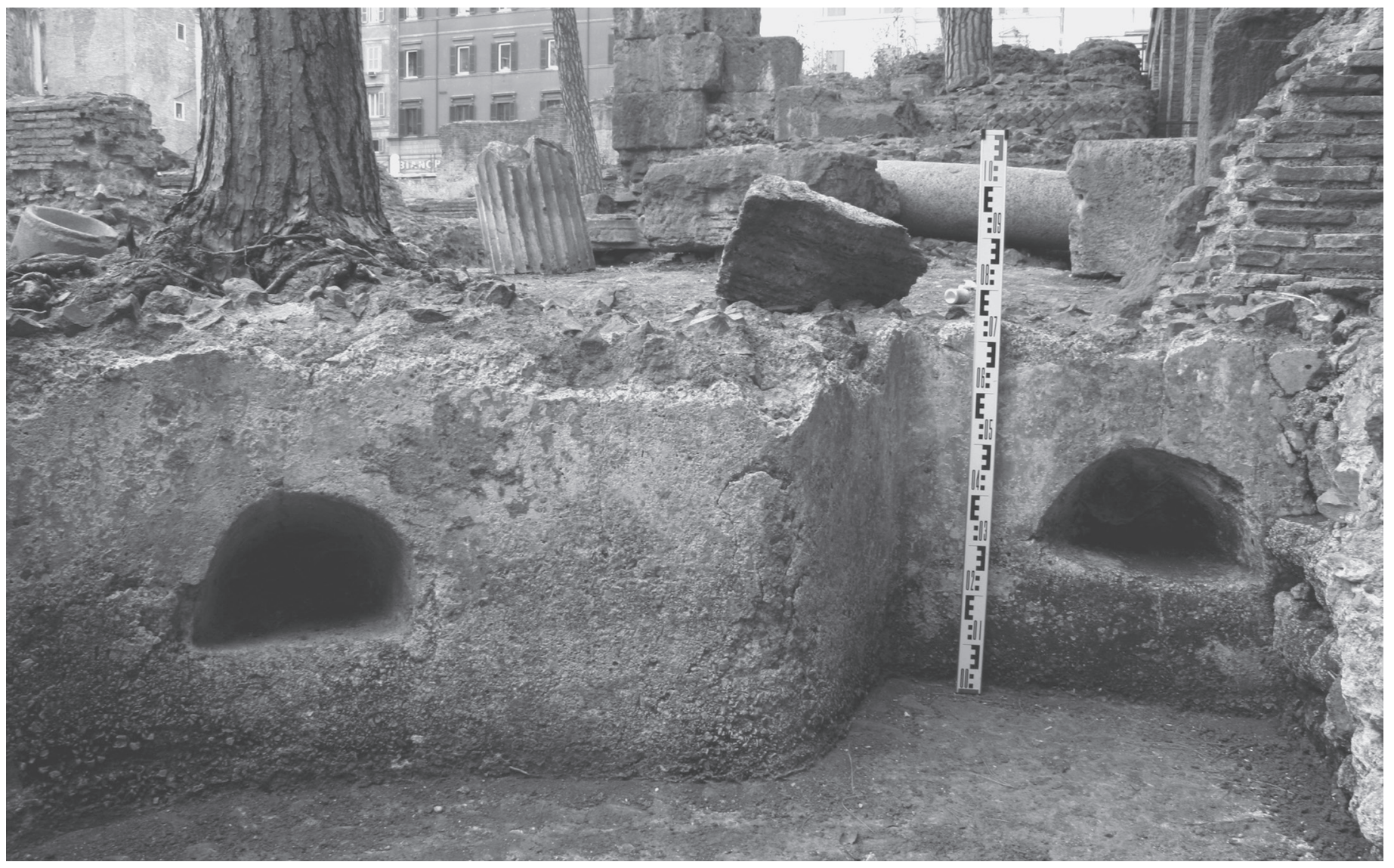

Fig. 8 - Area Sacra di largo Argentina. La vasca 1 con l'elemento centrale ancora visibile (Archivio Fotografico Sovrintendenza Capitolina- U.O. Monumenti di Roma).

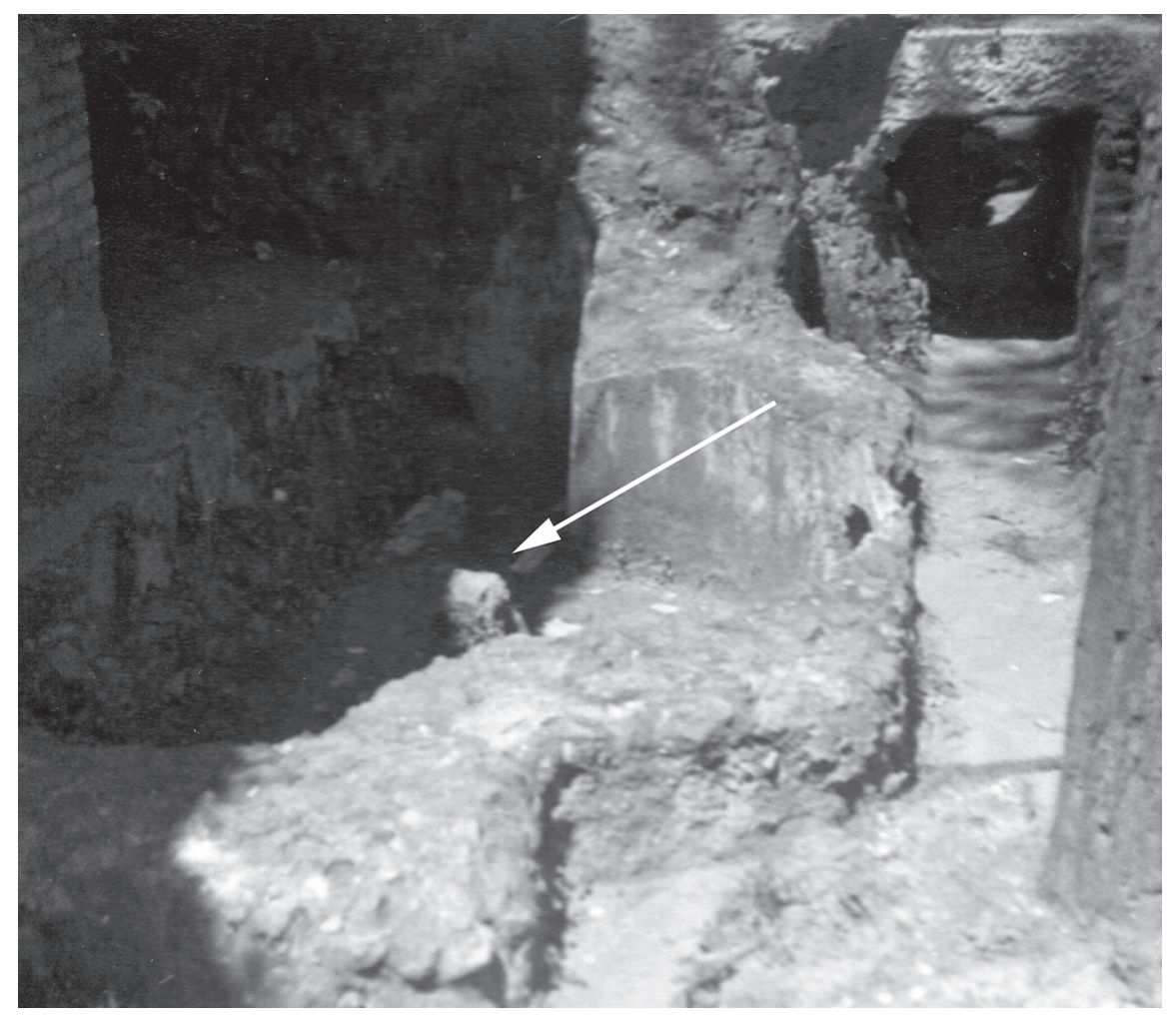


Fig. 9 - Area Sacra di largo Argentina. La vasca 4 al momento dello scavo. Sono chiaramente visibili la fistula di adduzione sul lato settentrionale, a destra nella foto, e lo scarico sul fondo (Archivio Fotografico Sovrintendenza Capitolina- U.O. Monumenti di Roma).

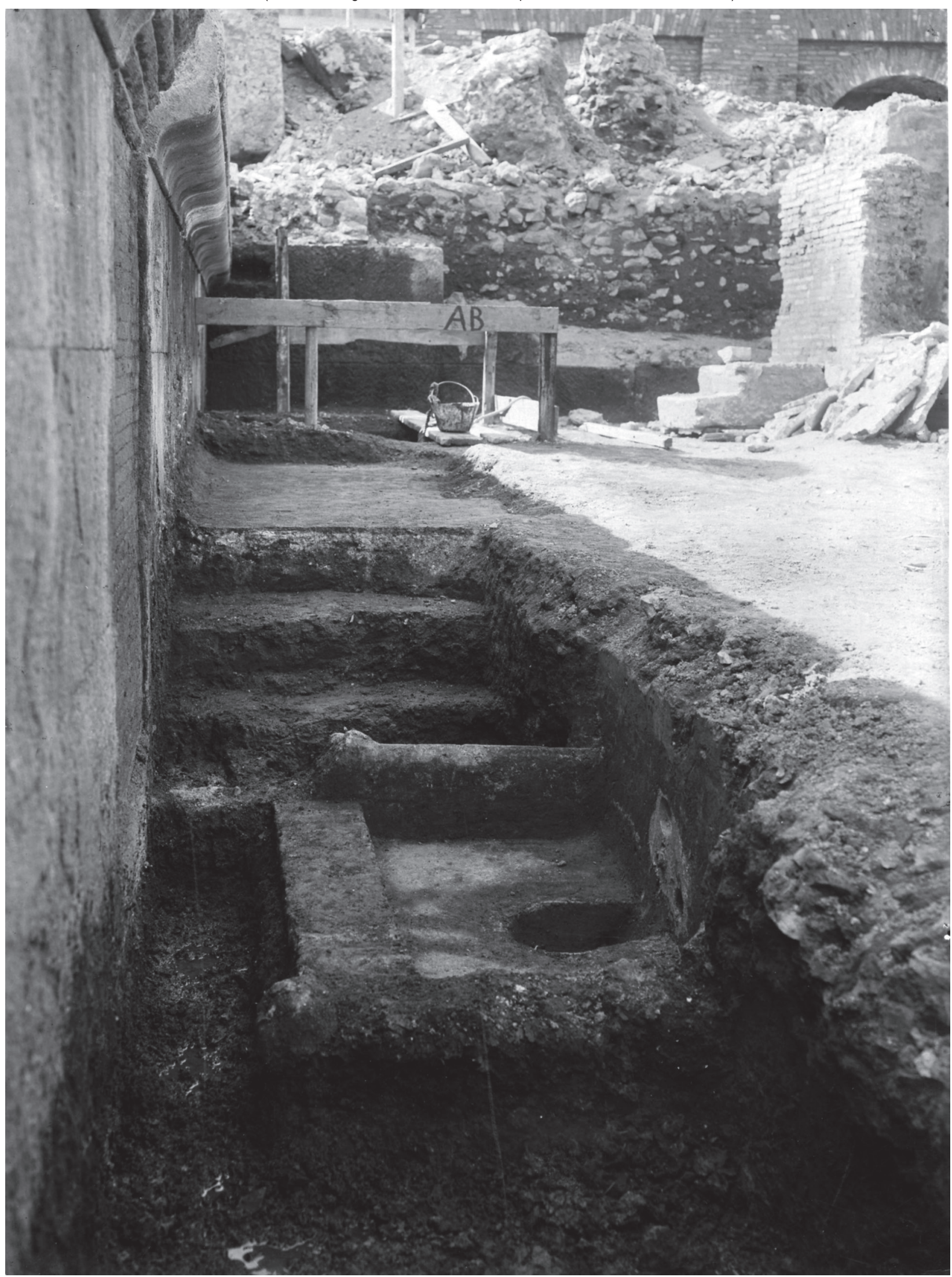


Fig. 10 - Area Sacra di largo Argentina. Particolare del conglomerato cementizio (vasca 3) utilizzato nella costruzione delle vasche mistilinee 1-3.

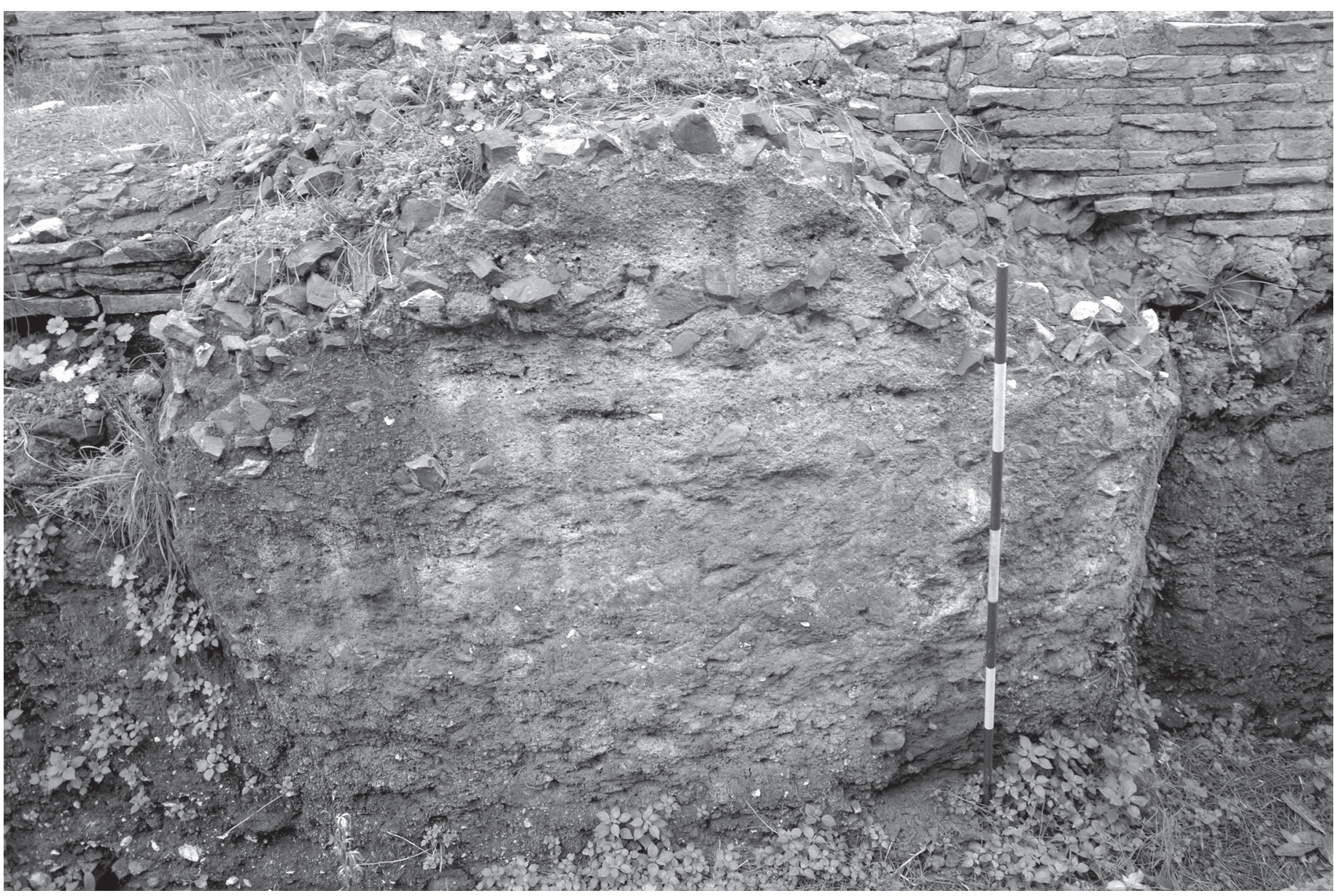

Tuttavia dal riesame di una fotografia di scavo degli anni '30 (Fig. 9), in cui si osserva la presenza di una fistula di alimentazione e di una fossetta posta sul fondo, è stato possibile individuare una sistemazione della vasca 4 riferibile ad una fase più tarda. Tale struttura idrica, di cui Marchetti Longhi ha lasciato una descrizione piuttosto oscura ${ }^{12}$, fu certamente riutilizzata tra la fine del V e il VI secolo d.C. quando l'area venne occupata da un monastero identificato con il Monasterium Boetianum. Alimentato da una fistula plumbea che vi conduceva l'acqua da una sorgente posta nei pressi dell'attuale piazza Paganica, l'impianto fu con ogni probabilità in uso fino a tutto il medioevo ${ }^{13}$.

\section{Osservazioni sulle tecniche costruttive}

\section{La vasca 5}

La vasca, oggi parzialmente interrata, è realizzata in opera cementizia con scapoli di tufo di forma e distribuzione irregolare legati con una malta piuttosto tenace di

12 Marchetti Longhi, 1971: 30.

13 Santangeli Valenzani 1994: 68, 70, Fig. 10. colore rossastro. Sulle pareti lo strato di rivestimento è scarsamente conservato, mentre è più consistente quello impermeabilizzante del fondo caratterizzato da un livello di cocciopesto ${ }^{14}$ di colore rosato (Fig. 2) e provvisto degli appositi rigonfiamenti arrotondati per evitare infiltrazioni e perdite d'acqua ${ }^{15}$.

Il campione, prelevato dal fondo, mostra una composizione costituita completamente da cocciopesto (calce e frammenti di terracotta) e non si rileva la presenza di pozzolana nell'impasto. Inoltre il rapporto legante/aggregato è 1:2 ${ }^{16}$.

A Roma gli esempi più antichi dell'inserzione di materiale fittile nei cementizi pavimentali (c.d. signina) sono documentati archeologicamente nelle domus poste alle pendici nordorientali del Palatino, databili alla fine del III secolo - inizi II secolo a.C. ${ }^{17}$ Esempi analoghi, inquadrabili

\footnotetext{
14 Per comodità si utilizza la denominazione di cocciopesto, sebbene sia nota l'inesattezza di questa terminologia; v. Grandi Carletti 2001: 183-197.

15 Bodon, Riera, Zanovello 1994: 311.

16 v. appendice.

17 Bragantini 2001: 12; Papi 1995: 337-352.
} 
nella prima metà del II secolo a.C., provengono dalle domus repubblicane di Fregellae ${ }^{18}$.

Per quanta riguarda l'utilizzo del cocciopesto come rivestimento idraulico il caso più antico conosciuto a Roma è rappresentato dal rivestimento delle pareti in opera incerta del Lacus Iuturnae, messe in relazione con gli interventi di Emilio Paolo dopo la battaglia di Pidna del 168 a.C. ${ }^{19}$

\section{Le vasche 1, 2, 3}

Le vasche 1,2 e 3 sono state tutte realizzate con la stessa tecnica costruttiva in conglomerato cementizio ricco di scaglie di selce, gettato in cavo libero con pareti e fondo privi di paramento (Fig. 10).

La malta di allettamento è costituita da calce e pozzolana per lo più rossa: in particolare nella vasca 1 è presente esclusivamente pozzolana rossa, mentre nelle altre due è prevalentemente rossa con una piccola percentuale di pozzolana nera. Il rivestimento in cocciopesto risulta ben conservato, ha uno spessore variabile fra i 2 ed i 4 centimetri e la superficie è allisciata con una calce più fine. Nella vasca 3 sono stati individuati tre strati di applicazione di cui il più esterno presenta una granulometria più fine rispetto ai primi due. Nella miscela, oltre a frammenti di terracotta di diversa granulometria e provenienza, sono presenti pozzolana e frammenti di selce. Il rapporto legante/aggregato evidenziato dall'analisi termogravimetrica è mediamente di 1:320.

Giuseppe Lugli riporta alcune osservazioni di carattere tecnico che si riscontrano puntualmente nelle vasche mistilinee $^{21}$. In primo luogo ne mette in evidenza l'utilizzo pressoché esclusivo in strutture con funzione di raccolta e contenimento dell'acqua quali cisterne, vasche o fontane; inoltre, rileva una serie di caratteristiche costruttive che si possono osservare anche nelle vasche 1, 2 e 3 dell'Area Sacra di largo Argentina, come l'impiego esclusivo di scapoli di selce per le cisterne d'acqua, l'assenza di paramento per favorire l'adesione dello strato di malta idraulica, l'utilizzo della stessa pietra con funzione di caementa tanto per le

\footnotetext{
18 Coarelli 1995: 17-30.

19 Steinby 1996: 169.

20 v. appendice.

21 L'autore segnala l'esempio della cisterna sul Palatino dietro la Casa di Livia (Lugli 1957: 415).
}

pareti che per il fondo - con l'unica differenza che gli scapoli adoperati in fondazione sono di volume maggiore e allettati in malta più abbondante e più magra - e, infine, la gettata del cementizio in cavo continuo poco profondo e non sbatacchiato.

È facilmente osservabile che le caratteristiche delineate corrispondono alla descrizione fornita da Vitruvio per l'opus signinum $^{22}$. In questa sede non ci si soffermerà sull'annoso dibattito circa l'uso del termine ${ }^{23}$, si intende, invece, mettere in evidenzia la stretta analogia tra la ricetta vitruviana dell'opera signina e la tecnica costruttiva impiegata per le vasche dell'Area Sacra di largo Argentina. La miscela prescritta da Vitruvio doveva essere gettata in cavo libero sia per la realizzazione delle pareti sia per il piano basale della cisterna, doveva inoltre essere pestata per mezzo di mazzaranghe - l'azione delle quali garantisce coesione e consistenza al materiale - ed essere costituita da calce molto forte, arena granulosa e pura nel rapporto di 5:2. Il pietrame duro (silex), infine, non doveva pesare più di una libra (g 327). La pezzatura dei caementa corrisponde esattamente a quella delle scaglie di leucitite che costituiscono lo scheletro del conglomerato delle vasche 1, 2 e 3 di largo Argentina, così come il rapporto 5:2 prescritto da Vitruvio è assai vicino a quello di 1:3 riscontrato dalle analisi. Rispettata, infine, anche la disposizione vitruviana circa la gettata di tale miscela in cavo libero.

Se l'attribuzione all'età augustea, proposta per il sistema di vasche absidate, è corretta, tali impianti, coevi al trattato di Vitruvio e realizzati secondo i suoi dettami, si inseriscono a pieno tra le opere realizzate in un periodo fondamentale per la sistemazione del Campo Marzio. Le profonde trasformazioni, legate alla figura di Agrippa, prevedevano, infatti, anche un importante riassetto idraulico del quartiere, quali la costruzione delle prime grandi terme pubbliche, l'annesso stagnum e la loro alimentazione per mezzo dell'Aqua Virgo. L'abbondanza di acque consentiva la creazione di spazi verdi e di luoghi ameni, che non si può escludere fossero legati al culto di divinità.

22 Vitr.VIII 6, 14-15: Sin autem loca dura erunt aut nimium venae penitus fuerint, tunc signinis operibus ex tectis aut superioribus locis excipiendae sunt copiae. In signinis autem operibus haec sunt facienda. Uti harena primum purissima asperrimaque paretur, caementum de silice frangatur ne gravius quam librarium, calce quam vehementissima mortario mixta ita ut quinque partes harenae ad duas respondeant. Eorum fossa ad libramentum altitudinis quod est futurum calcetur vectibus ligneis ferratis. Parietibus calcatis in medio quod erit terrenum exinaniatur ad libramentum infimum parietum. Hoc exaequato solum calcetur ad crassitudinem quae constituta fuerit. Ea autem si duplicia aut triplicia facta fuerint uti percolationibus transmutari possit, multo salubriorem et suaviorem aquae usum efficient.

23 Grandi Carletti 2001: 183-197; Gros 2003: 142-152; Gros 2006: 473-484. Di recente l'argomento è stato ripreso da P. Braconi (2009: 371-383). 


\section{Appendice: Caratterizzazione delle malte degli impianti idrici dell'Area Sacra di largo Argentina}

Appendice di

Maria Laura SANTARELLI, Maria Paola BRACCIALE, Alessandra BROGGI

Sapienza Università di Roma - CISTeC - Centro di Ricerca, Scienza e Tecnica per la Conservazione

del Patrimonio Storico-Architettonico

\section{Introduzione}

Lo studio riguarda la caratterizzazione di campioni di malte e intonaci a cocciopesto prelevati nelle vasche 1,2, 3, 5 dell'area sacra di largo Argentina.

I campioni sono stati analizzati con il microscopio ottico (MO) a diversi ingrandimenti in sezione spessa per valutare la tipologia di aggregato e il colore nel caso di malte pozzolaniche. Sono stati poi analizzati mediante analisi termogravimetrica (TGA) combinata con la calorimetria a scansione differenziale (DSC) per l'analisi qualitativa e quantitativa del legante e dell'aggregato per stabilire il rapporto legante/aggregato.

I risultati sono poi stati confrontati con la banca dati relativa alla caratterizzazione di malte di epoca romana che il CISTeC nei suoi 20 anni di attività ha raccolto a Roma.

\section{Campionatura}

\begin{tabular}{|c|c|}
\hline Campione & Descrizione \\
\hline 1 & Vasca 1_Intonaco a cocciopesto interno alla vasca \\
\hline 2 & Vasca 1_Intonaco a cocciopesto interno alla vasca \\
\hline 3 & Vasca 1_Malta del conglomerato cementizio \\
\hline 4 & Vasca 1_Malta sul pavimento della vasca \\
\hline 5 & $\begin{array}{c}\text { Vasca 2_Strati di malta: 5-1_Strato più interno; } \\
\text { 5-2_Strato intermedio; 5-3_Intonaco esterno }\end{array}$ \\
\hline 6 & Vasca 3_Intonaco a cocciopesto interno alla vasca \\
\hline 7 & Vasca 3_Incrostazione superiore al Camp.6 \\
\hline 8 & Vasca 3_Malta del conglomerato cementizio \\
\hline 9 & Vasca 2_Malta del conglomerato cementizio \\
\hline 10 & Grande Latrina_Intonaco a cocciopesto \\
\hline 11 & Vasca 5_Cocciopesto dalla pavimentazione \\
\hline
\end{tabular}

Il campione 5 presentava diverse stratificazioni ed è stato prelevato con 3 sotto-campioni, numerando gli strati dall'interno verso l'esterno.
Il campione 6 si è frammentato nel trasporto e si è deciso di analizzare tutti i frammenti, nominandoli Campione 6_sotto e Campione 6_sopra.

\section{Risultati delle analisi effettuate}

\section{Intonaci}

Tutti gli intonaci sono a cocciopesto (calce + frammenti di terracotta), ma bisogna rilevare una differenza di composizione. Nelle vasche 1, 2 e 3 nella miscela sono presenti, oltre a frammenti di terracotta a diversa granulometria e di diversa provenienza (da mattoni e/o tegole e da manufatti più raffinati), pozzolana e frammenti di selce.

Il rapporto legante/aggregato, evidenziato mediante l'analisi termogravimetrica, è mediamente di 1:3. Le differenze riscontrate sono da attribuirsi al degrado: i campioni sono stati prelevati, infatti, vicino alla superficie e dunque possono aver subito fenomeni di erosione che hanno modificato sensibilmente il rapporto.

Nella vasca 3 si sono individuati 3 strati di applicazione. Il più esterno presentava una granulometria più fine rispetto ai primi due.

Nota bene: il quarzo individuato dagli XRD non è da attribuirsi all'aggregato della malta, ma allo smagrante utilizzato nella composizione dei frammenti di cocciopesto.

\section{Pavimentazioni}

La pavimentazione della vasca 1 presenta composizione e rapporto legante/aggregato analoghi a quelli individuati negli intonaci corrispondenti. La vasca 5, invece, mostra una composizione costituita completamente da cocciopesto (calce e frammenti di terracotta) e non si rileva la presenza di pozzolana nell'impasto. Inoltre il rapporto legante/aggregato è 1:2.

\section{Malte di allettamento}

Le malte di allettamento della struttura delle vasche 1, 2 e 3 sono costituite da calce, frammenti di selce e pozzolana prevalentemente rossa (nella vasca 1 la pozzolana è totalmente rossa, nelle altre è prevalentemente rossa con una piccola parte di pozzolana nera). Il conglomerato cementizio in tutte le vasche è costituito da malta pozzolanica e pezzame di selce. 
Il rapporto legante/aggregato è 1:3 (i valori più alti sono dovuti a campioni più degradati).

Le vasche 1,2 e 3 sono state realizzate tutte con la stessa tecnica costruttiva. Il conglomerato cementizio costituito da selce e malta pozzolanica rossa con un rapporto 1:3 fa pensare ad una struttura che doveva avere valori di resistenza meccanica elevata. Anche il cocciopesto ha una composizione particolare in quanto realizzato con una componente pozzolanica mentre di norma per il cocciopesto viene usato solo materiale di terracotta (mattoni, tegole, elementi decorativi vari) che garantisce una reazione idraulica, ma lenta nel tempo. Questo composto sviluppa resistenze meccaniche in maniera molto graduale e non particolarmente forte, ma è adatto per la sua compattezza e bassa porosità per un'applicazione di impermeabilizzazione e in presenza di acqua. La presenza di pozzolana nell'impasto nei casi analizzati indica la volontà di garantire anche nell'intonaco e nel pavimento una migliore resistenza meccanica e una maggior rapidità di presa dell'intonaco oltre l'impermeabilità. Il rapporto legante aggregato di 1:3 per un intonaco non è molto usuale (di solito è 1:1 o 1:2), ma conferma la volontà di avere un intonaco anche più stabile e tenace oltre che impermeabilizzante, trattandolo più come una malta, che come un intonaco.

Il campione della vasca 5 invece è totalmente a cocciopesto e con rapporto 1:2 più caratteristico di un intonaco e/o di una pavimentazione. Questa considerazione andrebbe però confermata con la campionatura dell'intonaco vero e proprio della vasca, troppo degradato per fornire dati attendibili.

\section{Bibliografia}

BODON, RIERA, ZANOVELLO 1994: G. Bodon, I. Riera, P. Zanovello, Utilitas necessaria: sistemi idraulici nell'Italia romana, GEIE Progetto Quarta Dimensione, Milano, 1994.

BRACONI 2009: P. Braconi, Ostracus, asterico e lastrico. I pavimenti in cocciopesto degli antichi e l'opus signinum dei moderni, in C. Angelelli (ed.), Atti del XIV Colloquio dell'Associazione italiana per lo studio e la conservazione del mosaico, Spoleto 7-8 febbraio 2008, AISCOM - Colloqui dell'Associazione Italiana per lo Studio e la Conservazione del Mosaico (Scripta Manent - Tivoli), Tivoli, 2009, p. 371-383.

BRAGANTINI 2001: I. Bragantini, L'età repubblicana e augustea: nuove conoscenze dai colloqui AISCOM, in A. Paribeni (dir.), Atti del VII Colloquio Colloquio dell'Associazione italiana per lo studio e la conservazione del mosaico, Pompei 22-25 febbraio 2000, Ravenna, Edizioni del girasole, 2001, p. 11-19.

COARELLI 1981: F. Coarelli, L’Area Sacra di largo Argentina. Topografia e storia, in F. Coarelli, I. Kajanto, E.M. Steinby, L'Area Sacra di largo Argentina I, Roma, Quasar, 1981, p. 9-49.

COARELLI 1995: F. Coarelli, Gli scavi di Fregellae e la cronologia dei pavimenti repubblicani, in I. Bragantini, F. Guidobaldi (dir.), Atti del II Colloquio Colloquio dell'Associazione italiana per lo studio e la conservazione del mosaico, Roma dicembre 1994, Bordighera, Istituto Internazionale di Studi Liguri 1, 1995, p. 17-30.

DESSALES 2013: H. Dessales, Le partage de l'eau: fontaines et distribution hydraulique dans l'habitat urbain de l'Italie romaine, Roma, École française de Rome, 2013.

GALLUPPI 2007: R. Galluppi, La voragine insigne: Giuseppe Marchetti Longhi e la scoperta dell'area sacra del largo Argentina, Bullettino della Commissione Archeologica Comunale di Roma 108, 2007, p. 291-327.

GRANDI CARLETTI 2001: M. Grandi Carletti, Opus signinum e cocciopesto: alcune osservazioni terminologiche, in A. Paribeni (dir.), Atti del VII Colloquio Colloquio dell'Associazione italiana per lo studio e la conservazione del mosaico, Pompei 22-25 febbraio 2000, Ravenna, Edizioni del girasole, 2001, p. 183-197.

GROS 2003: P. Gros, L'opus signinum selon Vitruve dans la terminologie archéologique contemporaine, in G. Ciotta (ed.), Vitruvio nella cultura architettonica antica, medievale e moderna, Atti del convegno internazionale di Genova, 5-8 novembre 2001, Genova, De Ferrari, 2003, p. 142-152.

GROS 2006: P. Gros, Vitruve et la tradition des traités d'architecture: fabrica et ratiocinatio, Roma, École française de Rome, 2006.

LUGLI 1938: G. Lugli, I monumenti antichi di Roma e suburbio III, Roma, Giovanni Bardi Editore, 1938.

LUGLI 1957: G. Lugli, La tecnica edilizia romana con particolare riguardo a Roma e Lazio, Roma, Giovanni Bardi Editore, 1957.

MANACORDA, TAMASSIA 1985 : D. Manacorda, R. Tamassia, Il piccone del Regime, Roma, Curcio Editore, 1985.

MANCIOLI 1995 : D. Mancioli, L'Area Sacra Argentina, in L. Cardilli (dir.), Gli anni del Governatorato (1926-1944), Interventi urbanistici, scoperte archeologiche, arredo urbano, restauri, Roma, Kappa, 1985, p. 85-88.

MARCHETTI LONGHI 1970-71: G. Marchetti Longhi, Gli scavi dell'area sacra del Largo Argentina. Evoluzione e trasformazione dell'area dei templi dall'età imperiale all'inizio del Medio Evo, Bullettino della Commissione Archeologica Comunale di Roma 82, 1970-71, p. 7-62.

MESSA 1995: L. Messa, La demolizione dell'isolato di S. Nicola ai Cesarini e la scoperta dell'area Sacra Argentina, in L. Cardilli (dir.), Gli anni del Governatorato (1926-1944), Interventi urbanistici, scoperte archeologiche, arredo urbano, restauri, Roma, Kappa, 1985, p. 77-84.

PAPI 1995: E. Papi, I pavimenti delle domus della pendice settentrionale del Palatino (VI-II sec. a.C.), in I. Bragantini, F. Guidobaldi (dir.), Atti del II Colloquio Colloquio dell'Associazione italiana per lo studio e la conservazione del mosaico, Roma 5-7 dicembre 1994, Bordighera, Istituto Internazionale di Studi Liguri, 1, 1995, p. 337-352.

SANTANGELI VALENZANI 1994: R. Santangeli Valenzani, Tra la Porticus Minucia e il Calcarario. L'area sacra di largo Argentina nell'alto medioevo, Archeologia Medievale 21, 1994, p. 57-98.

STEINBY 1981 : E. M. Steinby, I bolli laterizi dell'Area Sacra di largo Argentina, in F. Coarelli , I. Kajanto, E.M. Steinby, L'Area Sacra di largo Argentina I, Roma, Quasar, 1981, p. 297-332.

STEINBY 1996 : E. M. Steinby, s.v. Lacus Iuturnae, in E. M. Steinby (dir.), Lexicon Topographicum Urbis Romae, Roma, Quasar, 1996, p. $168-170$. 


\section{Table des matières}

Introduction - Les mortiers antiques, un champ d'investigation en essor 5

SOphie BOUFFIER

Connecting materials, connecting cultures. Le malte idrauliche nell'Opus agriculturae

di Palladio: compendio di un millenario know-how mediterraneo

Antonello V. GRECO

L'hydraulicité des mortiers antiques, entre préconçus et réalité : l'exemple des mortiers de tuileau et autres matériaux de Gaule romaine

Arnaud COUTELAS

Pozzolans in Mortar in the Roman Empire: An Overview and Thoughts on Future Work 31 Lynne C. LANCASTER

Pre-Roman waterproofing mortars in Ancient Corinth: materials and technology 41

Ruth SIDDALL

Des mortiers hydrauliques dans la ville grecque de Marseille? IV ${ }^{e}$ siècle av. J.-C. - 50 av. J.-C. 53 Un état des recherches Anne-Marie D’Ovidio, Philippe BROMBLET

The Concrete Floors of Megara Hyblaea. A Sicilian Perspective 75 Frédéric MÈGE

Analisi e rilettura di alcuni impianti idrici nell'Area Sacra di largo Argentina 87 Monica CECI, Alessandra MARCHELLO, Marcello TURCI

Appendice di Maria Laura SANTARELLI, Maria Paola BRACCIALE, Alessandra BROGGI

Hydraulic Mortars in the Imperial Residence. An Interdisciplinary Project

on the Palatine/Rome 99

Andrea SCHMÖLDER-VEIT, Laura THIEMANN with contributions from Felix HENKE and Frank SCHLÜTTER 
Rivestire e proteggere le pareti degli edifici in epoca romana. I casi dei magazzini di Roma, Ostia e Portus

Milena MIMMO, Mathilde CARRIVE

Mortiers et bétons de tuileaux dans les aqueducs romains : le cas des aqueducs d'Arles et de Barbegal

Philippe LEVEAU

Elaborazioni tecniche dell'opera cementizia nel "Lazio del Calcare" nella tarda età repubblicana: l'opus signinum e Segni 145

Francesco Maria CIFARELLI, Christopher J. SMITH, Federica COLAIACOMO, Stephen J. KAY, Letizia CECCARELLI, Camilla PANZIERI

Le rôle des mortiers antiques dans la gestion de l'eau: complexités et défis 167 Remarques conclusives

Iván FUMADÓ ORTEGA

Index 173 


\section{Mortiers et hydraulique}

\section{en Méditerranée antique}

ARCHÉOLOGIES MÉDITERRANÉENNES

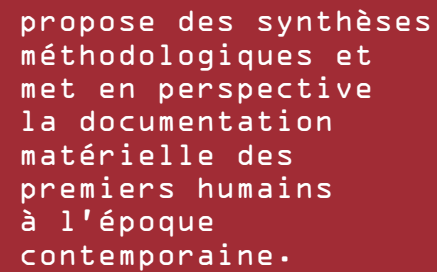

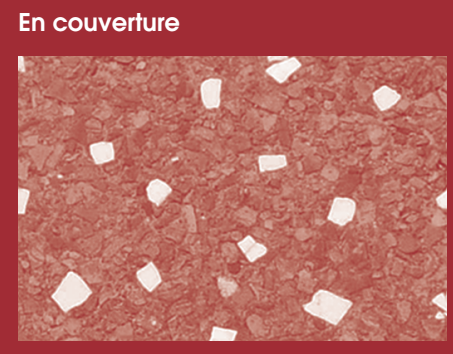

Pavement mosaiqué de maison, IV / III siècles av. J.-C., Kerkouane (Tunisie). Photographie : I. Fumadó Ortega, 2007
Les sociétés antiques ont souvent imperméabilisé leurs installations hydrauliques par des mortiers, qu'il s'agisse de citernes, de canalisations, de fontaines ou de thermes, mais la recherche archéologique ne s'est emparée de cette problématique qu'à une époque récente. Quelle était la composition de ces mortiers qualifiés à tort d'hydrauliques? Celle-ci variait-elle selon les époques, selon les régions, selon les maîtres d'ouvrage ou types d'aménagements? Quelles étaient les techniques de fabrication? Quid de leurs modes d'application? Peut-on les dater et ainsi préciser leur durée et période d'utilisation? Autant de questions que cet ouvrage veut aborder à partir d'une rencontre tenue à la Maison méditerranée des sciences de I'homme à Aix-en-Provence en 2016, dans le cadre du réseau HYDR@MED. Archéologues, ingénieurs, chimistes, restaurateurs, et historiens explorent ici les rapports étroits que I'hydraulique antique a entretenus avec les mortiers de chaux pour répondre à la demande parfois démesurée des usagers et optimiser l'efficacité des aménagements dans des milieux naturels souvent pauvres en eau. Ils mettent ainsi l'accent sur l'hétérogénéité et la diversité des choix et méthodes mis en œuvre par les Anciens pour conserver leur eau dans les meilleures conditions. Si les études sur les mortiers utilisés dans les aménagements hydrauliques antiques suscitent de plus en plus d'attention, leur analyse archéométrique fournit un nombre de données toujours plus exhaustif et spécialisé. Pour mettre en lumière des questions transdisciplinaires et des dynamiques sur la longue durée, notre ouvrage entend contextualiser les textes et recettes transmis par la littérature antique et les comparer à la réalité des enduits de la Corinthe grecque, du Palatin romain, ou des sols de quelques villes grecques, mis au jour par l'archéologie.

Iván Fumadó Ortega, ancien élève de l'École espagnole d'histoire et d'archéologie de Rome, est chercheur Ramón y Cajal à l'Université de Valence. Spécialiste du phénomène urbain des sociétés phénicopuniques, il s'intéresse aux transferts culturels en Méditerranée occidentale préromaine.

Sophie Bouffier, ancienne élève de l'École normale supérieure, est professeur d'histoire grecque à l'Université d'Aix-Marseille. Spécialiste de l'expansion grecque en Méditerranée occidentale, elle a consacré une grande partie de ses travaux à l'étude des modes de gestion des ressources hydriques en Sicile et Gaule méridionale.

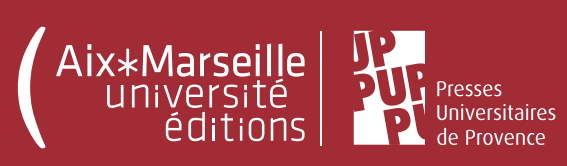

A* A*Midex

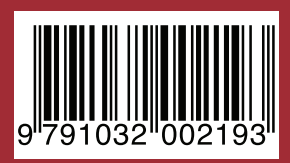

